From the Dana-Farber Cancer Institute, Boston, MA; Mayo Clinic, Rochester; Minnesota Oncology Hematology, P.A., Minneapolis, MN; St Vincent's Comprehensive Cancer Center, New York, NY; Duke University Medical Center, Durham; University of North Carolina at Chapel Hill, Chapel Hill, NC; VA Pittsburgh Healthcare System, Pittsburgh, PA; and University of Nebraska Medical Center, Omaha, NE.

Submitted April 11, 2007; accepted April 11, 2007; published online ahead of print at www.jco.org on May 21, 2007.

Authors' disclosures of potential conflicts of interest and author contributions are found at the end of this article.

Address reprint requests to the American Society of Clinical Oncology, 1900 Duke St, Ste 200, Alexandria, VA 22314; e-mail: guidelines@asco.org.

C 2007 by American Society of Clinical Oncology

0732-183X/07/2517-1/\$20.00

DOI: 10.1200/JCO.2007.12.1269

\title{
American Society of Clinical Oncology 2007 Clinical Practice Guideline Update on the Role of Bisphosphonates in Multiple Myeloma
}

Robert A. Kyle, Gary C. Yee, Mark R. Somerfield, Patrick J. Flynn, Susan Halabi, Sundar Jagannath, Robert Z. Orlowski, David G. Roodman, Patricia Twilde, and Kenneth Anderson

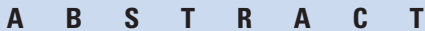

\section{Purpose}

To update the recommendations for the use of bisphosphonates in the prevention and treatment of bone disease in multiple myeloma. The Update Committee expanded the guideline to include a discussion of osteonecrosis of the jaw (ONJ).

\section{Methods}

For the 2007 update, an Update Committee composed of members from the full panel completed a review and analysis of data published since 2002. Searches of Medline and the Cochrane Collaboration Library databases were performed.

\section{Recommendations}

For multiple myeloma patients who have, on plain radiograph(s) or imaging studies, lytic destruction of bone or spine compression fracture from osteopenia, intravenous pamidronate 90 mg delivered over at least 2 hours or zoledronic acid 4 mg delivered over at least 15 minutes every 3 to 4 weeks is recommended. Clodronate is an alternative bisphosphonate approved worldwide, except in the United States, for oral or intravenous administration. New dosing guidelines for patients with pre-existing renal impairment were added to the zoledronic acid package insert. Although no similar dosing guidelines are available for pamidronate, the Update Committee recommends that clinicians consider reducing the initial pamidronate dose in patients with pre-existing renal impairment. Zoledronic acid has not been studied in patients with severe renal impairment and is not recommended in this setting. The Update Committee suggests that bisphosphonate treatment continue for a period of 2 years. At 2 years, physicians should seriously consider discontinuing bisphosphonates in patients with responsive or stable disease, but further use is at the discretion of the treating physician. The Update Committee also discusses measures regarding ONJ.

\section{J Clin Oncol 25. (C) 2007 by American Society of Clinical Oncology}

\section{INTRODUCTION}

The American Society of Clinical Oncology (ASCO) first published evidence-based clinical practice guidelines on the role of bisphosphonates in multiple myeloma in 2002. ASCO guidelines are updated at intervals by an Update Committee of the original Expert Panel. The Update Committee has expanded the scope of the guidelines to include recommendations concerning the association of osteonecrosis of the jaw (ONJ) and bisphosphonate therapy (Table 1 provides a summary of the guideline recommendations).

\section{UPDATE METHODOLOGY}

For the 2007 update, an Update Committee composed of members from the full Expert Panel (Ap- pendix 1) was formed to complete the review and analysis of data published since 2002. The Update Committee's literature review focused attention on available randomized clinical trials, clinical practice guidelines, and systematic reviews of published clinical trials and a meta-analysis report. For the guideline recommendations related to ONJ, the Update Committee considered data and reports from manufacturers of bisphosphonates, governmental agencies, and other dental and medical professional societies. Details of the literature searches are provided in Appendix 2.

The Update Committee held a single face-toface meeting to consider the evidence for each of the 2007 recommendations. Additional work to complete the update was completed via teleconferences with the steering group and with the full Update Committee. Representatives from industry attended 
Table 1. Summary of Recommendations

Specific Recommendations

Lytic disease on plain radiographs or imaging studies

Monitoring
2007 Recommendations

For multiple myeloma patients who have, on plain radiograph(s) or imaging studies, lytic destruction of bone or compression fracture of the spine from osteopenia, intravenous pamidronate $90 \mathrm{mg}$ delivered over at least 2 hours or zoledronic acid $4 \mathrm{mg}$ delivered over at least 15 minutes every 3 to 4 weeks is recommended. In light of data from Zervas et al ${ }^{1}$ showing a 9.5-fold greater risk for the development of osteonecrosis of the jaw with zoledronic acid compared with pamidronate, patients may prefer pamidronate to zoledronic acid until more data become available on this adverse effect of bisphosphonate therapy. Clodronate is an alternative bisphosphonate approved worldwide, except in the United States, for either oral or intravenous administration.

As a result of increased concerns over renal adverse events, new dosing guidelines for patients with pre-existing renal impairment were added to the zoledronic acid package insert. The new guidelines recommend that patients with pre-existing mild-to-moderate renal impairment (estimated creatinine clearance, 30 to $60 \mathrm{~mL} / \mathrm{min}$ ) should receive a reduced dosage of zoledronic acid. No changes in infusion time or interval are required. Zoledronic acid has not been studied in patients with severe renal impairment and is not recommended for use in these patients. Pamidronate $90 \mathrm{mg}$ administered over 4 to 6 hours is recommended for patients with extensive bone disease and existing severe renal impairment (serum creatinine level $>3.0 \mathrm{mg} / \mathrm{dL}[265 \mu \mathrm{mol} / \mathrm{L}]$ or an estimated creatinine clearance $<30 \mathrm{~mL} / \mathrm{min}$ ). Although no dosing guidelines are available for patients with pre-existing renal impairment, the Update Committee recommends that clinicians consider reducing the initial pamidronate dose in that setting. Infusion times less than 2 hours with pamidronate or less than 15 minutes with zoledronic acid should be avoided. The Update Committee recommends that serum creatinine should be monitored before each dose of pamidronate or zoledronic acid, in accordance with FDA-approved labeling. In patients who develop renal deterioration without apparent cause during bisphosphonate therapy, zoledronic acid or pamidronate should be withheld Bisphosphonate therapy can be resumed, at the same dosage as that before treatment interruption, when the serum creatinine returns to within $10 \%$ of the baseline level. Serum calcium, electrolytes, phosphate, magnesium, and hematocrit/hemoglobin should also be monitored regularly, although there is no evidence on which to base a recommendation for time intervals. The Update Committee also recommends intermittent evaluation (every 3 to 6 months) of all patients receiving pamidronate or zoledronic acid therapy for the presence of albuminuria. In patients experiencing unexplained albuminuria (defined as $>500 \mathrm{mg} / 24$ hours of urinary albumin), discontinuation of the drug is advised until the renal problems are resolved. These patients should be reassessed every 3 to 4 weeks (with a 24-hour urine collection for total protein and urine protein electrophoresis), and pamidronate should be reinstituted over a longer infusion time ( $\geq 4$ hours) and at doses not to exceed 90 mg every 4 weeks when the renal function returns to baseline. The Update Committee supports the use of screening urinalysis for proteinuria but underscores that a 24-hour urine collection for determination of total protein and electrophoresis is required if the test is positive. Although no similar guidelines are available for zoledronic acid, some Update Committee members recommend that zoledronic acid be reinstituted over a longer infusion time ( $\geq 30$ minutes)

Duration of therapy

A single randomized clinical trial has shown no benefit of monthly bisphosphonates after a tandem transplantation. ${ }^{2}$ There was no difference in the proportion of skeletal events in the pamidronate-containing regimens (21\% and $18 \%)$ compared with no maintenance $(24 \%)$ after 29 months of follow-up. The Update Committee suggests that therapy with bisphosphonates be administered monthly for a period 2 years. (The trial by Attal et al ${ }^{2}$ suggests 1 year if the patient is in a CR or NCR after a tandem transplantation.) At 2 years, the physician should seriously consider stopping bisphosphonates in patients with responsive or stable disease, but their further use is at the discretion of the treating physician. There are no data to support a more precise recommendation for duration of bisphosphonate therapy in this group of patients. For those patients in whom bisphosphonates were withdrawn after 2 years, the drug should be resumed upon relapse with new-onset skeletal-related events.

There is no change from the original guideline recommendation. It is reasonable to start intravenous bisphosphonates in multiple myeloma patients with osteopenia but no radiographic evidence of lytic bone disease. Note, patients with nonlytic lesions have been included in selected trials but have not been the primary focus of the trial or of sufficient number to be separately analyzed.

There is no change from the original guideline recommendation. Starting bisphosphonates in patients with solitary plasmacytoma or smoldering (asymptomatic) or indolent myeloma is not recommended.

There is no change from the original guideline recommendation. Starting bisphosphonates in patients with monoclonal gammopathy of undetermined significance is not recommended.

There is no change from the original guideline recommendation The use of the biochemical markers of bone metabolism to monitor bisphosphonate use is not suggested for routine care

There is no change from the original guideline recommendation. Intravenous pamidronate or zoledronic acid is recommended for patients with pain as a result of osteolytic disease and as an adjunctive treatment for patients receiving radiation therapy, analgesics, or surgical intervention to stabilize fractures or impending fractures.

Osteonecrosis of the jaw*

Osteonecrosis of the jaw is an uncommon but potentially serious complication of intravenous bisphosphonates. The Update Committee agrees with the recommendations described in the revised FDA label for zoledronic acid and pamidronate, Dear Doctor letters, a white paper, and various position papers or statements. All cancer patients should receive a comprehensive dental examination and appropriate preventive dentistry before bisphosphonate therapy. Active oral infections should be treated, and sites at high risk for infection should be eliminated. While on therapy, patients should maintain excellent oral hygiene and avoid invasive dental procedures, if possible.

Abbreviations: FDA, Food and Drug Administration; CR, complete response; NCR, near complete response.

${ }^{*}$ This topic is new to the guideline. 
the Update Committee meeting and provided preprints and reprints of relevant studies. The guideline was circulated in draft form to the Update Committee. ASCO's Health Services Committee and the ASCO Board of Directors also reviewed the final document.

It important to emphasize that guidelines and technology assessments cannot always account for individual variation among patients. They are not intended to supplant physician judgment with respect to particular patients or special clinical situations and cannot be considered inclusive of all proper methods of care or exclusive of other treatments reasonably directed at obtaining the same result.

Accordingly, ASCO considers adherence to this guideline assessment to be voluntary, with the ultimate determination regarding its application to be made by the physician in light of each patient's individual circumstances. In addition, this guideline describes the use of procedures and therapies in clinical practice; it cannot be assumed to apply to the use of these interventions performed in the context of clinical trials, given that clinical studies are designed to evaluate or validate innovative approaches in a disease for which improved staging and treatment is needed. Because guideline development involves a review and synthesis of the latest literature, a practice guideline also serves to identify important questions and settings for further research.

\section{SUMMARY OF LITERATURE REVIEW RESULTS}

The literature search conducted for this update identified several relevant reports, including two articles reporting the results of randomized clinical trials, one clinical practice guideline, one consensus statement, ${ }^{3}$ and two systematic reviews of the literature. One of the randomized clinical trials identified evaluated the effect of pamidronate on skeletal events and disease progression in patients with earlystage, untreated multiple myeloma. ${ }^{4}$ A second report presented the results of a 25-month final analysis of a previously published randomized clinical trial that compared the safety and efficacy of pamidronate and zoledronic acid. ${ }^{5}$ The clinical practice guideline, which was developed by the Cancer Care Ontario Program in Evidence-Based Care, ${ }^{6}$ addressed the role of bisphosphonates in the management of skeletal complications for patients with multiple myeloma. ${ }^{6}$ This guideline was based in large part on the Cochrane Collaboration systematic review and meta-analysis conducted by Djulbegovic et al. ${ }^{7}$ The consensus statement was developed by a multidisciplinary panel at the Mayo Clinic that included hematologists, dental specialists, and nurses specializing in the treatment of multiple myeloma. The statement addressed the choice of a bisphosphonate for multiple myeloma patients, duration of therapy, and adverse events associated with bisphosphonate therapy. ${ }^{3}$ Finally, the systematic reviews analyzed the role of bisphosphonates in metastatic disease ${ }^{8}$ and the role of bisphos- phonates in the relief of pain secondary to bone metastases. ${ }^{9}$ The results of the two randomized clinical trials and the two systematic reviews are summarized in the relevant literature update and discussion sections that follow.

\section{GUIDELINE RECOMMENDATIONS}

\section{Lytic Disease on Plain Radiographs or Imaging}

2007 recommendation. For multiple myeloma patients who have, on plain radiograph(s) or imaging, lytic destruction of bone or compression fracture of the spine from osteopenia, intravenous pamidronate $90 \mathrm{mg}$ delivered over at least 2 hours or zoledronic acid $4 \mathrm{mg}$ delivered over at least 15 minutes every 3 to 4 weeks is recommended. In light of data from Zervas et $\mathrm{al}^{1}$ showing a 9.5-fold greater risk for the development of $\mathrm{ONJ}$ with zoledronic acid compared with pamidronate, patients may prefer pamidronate to zoledronic acid until more data become available on this adverse effect of bisphosphonate therapy. Clodronate is an alternative bisphosphonate that has been approved worldwide, except in the United States, for either oral or intravenous administration.

Literature update and discussion. The literature search for the 2007 guideline identified an updated analysis of a previously published randomized clinical trial. Rosen et $\mathrm{al}^{5}$ reported data from a large phase III randomized trial that compared 4- or 8-mg doses of zoledronic acid with $90 \mathrm{mg}$ of pamidronate every 3 to 4 weeks in patients with multiple myeloma or breast cancer who had lytic disease. ${ }^{10}$ After a follow-up period of 25 months, zoledronic acid reduced the overall proportion of patients with a skeletal-related event and reduced the skeletal morbidity to an extent similar to that for pamidronate. Compared with pamidronate, zoledronic acid reduced the overall risk of developing skeletal complications (including hypercalcemia) by an additional $16 \%(P=.03)$.

A systematic review of the literature (1966 to 2001) by Ross et al ${ }^{8}$ on the use of bisphosphonates in metastatic disease, including multiple myeloma, was published in 2004. There is considerable overlap between this review and the Cochrane Collaboration systematic review published by Djulbegovic et $\mathrm{al}^{7}$ in 2002 that was featured in the original ASCO guideline for bisphosphonates in multiple myeloma. Ross et $\mathrm{al}^{8}$ included nine of the 11 trials that were considered in the Cochrane Collaboration systematic review.

Ross et $\mathrm{al}^{8}$ pooled data from three randomized clinical trials with a total of 1,079 patients for vertebral fractures, combined fractures, and hypercalcemia skeletal morbidity outcomes. The results indicated that, compared with placebo, bisphosphonate therapy significantly reduced the risk of vertebral fractures but not combined fractures or hypercalcemia (Table 2). Combined analyses of the effect of

\begin{tabular}{|lccrr|}
\hline \multicolumn{4}{|c|}{ Table 2. Summary Statistics From Subgroup Analysis of Skeletal Morbidity End Points From Multiple Trials } \\
\hline Skeletal Morbidity Outcome & Odds Ratio & $95 \% \mathrm{Cl}$ & No. of Studies & No. of Patients \\
\hline Vertebral fractures: yes $v$ no & 0.583 & 0.419 to 0.812 & 2 & 913 \\
Combined fractures: yes $v$ no & 0.776 & 0.539 to 1.120 & 2 & 543 \\
Hypercalcemia: yes $v$ no & 0.968 & 0.687 to 1.365 & 3 & .001 \\
\hline
\end{tabular}

NOTE. Adapted with permission. ${ }^{8}$ 
bisphosphonates on survival outcomes were not reported for the multiple myeloma subset of the trials in the systematic review.

\section{Monitoring}

2007 recommendations. As a result of increased concerns over renal adverse events, new dosing guidelines for patients with preexisting renal impairment were added to the zoledronic acid package insert. The new guidelines recommend that patients with pre-existing mild-to-moderate renal impairment (estimated creatinine clearance, 30 to $60 \mathrm{~mL} / \mathrm{min}$ ) should receive a reduced dosage of zoledronic acid. No changes in infusion time or interval are required. Zoledronic acid has not been studied in patients with severe renal impairment and is not recommended for use in these patients. Pamidronate $90 \mathrm{mg}$ administered over 4 to 6 hours is recommended for patients with extensive bone disease and existing severe renal impairment (serum creatinine level $>3.0 \mathrm{mg} / \mathrm{dL}[265 \mu \mathrm{mol} / \mathrm{L}]$ or an estimated creatinine clearance $<30 \mathrm{~mL} / \mathrm{min}$ ). Although no dosing guidelines are available for patients with pre-existing renal impairment, the Update Committee recommends that clinicians consider reducing the initial pamidronate dose in that setting.

Infusion times less than 2 hours with pamidronate or less than 15 minutes with zoledronic acid should be avoided. The Update Committee recommends that serum creatinine should be monitored before each dose of pamidronate or zoledronic acid, in accordance with US Food and Drug Administration-approved labeling. In patients who develop renal deterioration with no other apparent cause during bisphosphonate therapy, zoledronic acid or pamidronate should be withheld. Bisphosphonate therapy can be resumed, at the same dosage as that before treatment interruption, when the serum creatinine returns to within $10 \%$ of the baseline level. Serum calcium, electrolytes, phosphate, magnesium, and hematocrit/hemoglobin should also be monitored regularly, although there is no evidence on which to base a recommendation for time intervals. The Update Committee also recommends intermittent evaluation (every 3 to 6 months) of all patients receiving pamidronate or zoledronic acid therapy for the presence of albuminuria. In patients experiencing unexplained albuminuria (defined as $>500 \mathrm{mg} / 24$ hours of urinary albumin), discontinuation of the drug is advised until the renal problems are resolved. When the proteinuria returns to baseline, these patients should be reassessed every 3 to 4 weeks (with a 24-hour urine collection for total protein and urine protein electrophoresis), and pamidronate should be reinstituted over a longer infusion time ( $\geq 4$ hours) and at doses not to exceed $90 \mathrm{mg}$ every 4 weeks. The Update Committee supports the use of screening urinalysis for proteinuria but underscores that a 24-hour urine collection for determination of total protein and electrophoresis is required if the screening test is positive. Although no similar guidelines are available for zoledronic acid, some Update Committee members recommend that zoledronic acid be reinstituted over a longer infusion time ( $\geq 30$ minutes).

Literature update and discussion. Zoledronic acid and pamidronate are associated with renal deterioration, particularly in patients with pre-existing renal impairment and in patients who receive multiple cycles of therapy. In several randomized comparisons of zoledronic acid ( $4 \mathrm{mg}$ as a 15-minute infusion) versus pamidronate (90 mg as a 2-hour infusion) or placebo, approximately $12 \%$ of patients with multiple myeloma, breast cancer, or other solid tumors developed evidence of renal deterioration during the study period (up to 24 months). In these studies, which involved more than 1,000 patients, deterioration of renal function was defined as a change in baseline serum creatinine $\geq 0.5 \mathrm{mg} / \mathrm{dL}$ or $\geq$ two times the baseline value in patients with a normal baseline serum creatinine level $(<1.4$ $\mathrm{mg} / \mathrm{dL}$ ) or a change from baseline serum creatinine $\geq 1.0 \mathrm{mg} / \mathrm{dL}$ or $\geq$ two times the baseline value in patients with an abnormal baseline serum creatinine $(\geq 1.4 \mathrm{mg} / \mathrm{dL})$. In the randomized comparison of zoledronic acid versus pamidronate, the incidence of renal deterioration was similar in the two groups. ${ }^{5}$ In the placebo-controlled trials, the risk of renal deterioration was higher in patients who received zoledronic acid compared with patients randomly assigned to the placebo group $\left(13.2 \% \vee 8.7 \%\right.$, respectively). ${ }^{5,11}$ In the subgroup of patients with abnormal serum creatinine, the risk seemed to be higher in the zoledronic acid group compared with the placebo group (23.8\% $v 10 \%$, respectively). In another analysis based on estimated creatinine clearance, patients in the zoledronic acid group with pre-existing moderate renal impairment (creatinine clearance of 30 to $49 \mathrm{~mL} / \mathrm{min}$ ) showed the highest risk of renal deterioration compared with the placebo group (32.1\% $v 7.7 \%$, respectively). ${ }^{12}$ No difference was observed in patients with pre-existing mild renal impairment (creatinine clearance of 50 to $69 \mathrm{~mL} / \mathrm{min}$ ) who received zoledronic acid compared with the placebo group ( $7.5 \% v 9.0 \%$, respectively). Given these observations, new dosing guidelines for patients with pre-existing renal impairment were added to the zoledronic acid package insert in January of 2005. These guidelines recommend a lower initial zoledronic acid dose (ranging from 3.0 to $3.5 \mathrm{mg}$ ) depending on the estimated creatinine clearance. The lower doses were calculated to achieve the same area under the curve as that achieved in patients with a creatinine clearance of $75 \mathrm{~mL} / \mathrm{min}$. Although no similar dosing guidelines are available for pamidronate, the Update Committee recommends that clinicians consider reducing the initial pamidronate dose in patients with pre-existing renal impairment.

Oral and intravenous ibandronate may have a different renal safety profile than pamidronate and zoledronic acid. In randomized placebo-controlled trials, the incidence of renal adverse effects with oral and intravenous ibandronate was similar to that observed with placebo (approximately 5\% in both groups). However, no definitive conclusions about the comparative safety of ibandronate versus zoledronic acid can be reached. Randomized head-to-head comparisons of ibandronate versus zoledronic acid are planned or ongoing.

Data on the long-term renal safety of bisphosphonates are limited. In one series of 57 patients with cancer who were treated with either pamidronate or pamidronate plus zoledronic acid every 3 to 4 weeks for more than 24 months (median, 34 months; range, $24+$ to $131+$ months), a notable increase in serum creatinine occurred in seven patients (12\%). ${ }^{13}$ Bisphosphonates were discontinued in one patient and continued in the remaining six patients; in the six patients who continued treatment, serum creatinine returned to normal in two patients and did not increase in the other four patients. In another series of 22 patients with cancer who were treated with intravenous bisphosphonates for more than 24 months, four patients (22\%) had a notable increase in serum creatinine. ${ }^{14}$ The renal safety of alternative dosing intervals (eg, every 12 weeks) of bisphosphonates is not known.

Renal deterioration can progress to renal failure and dialysis. ${ }^{15-20}$ Renal failure has occurred in patients with multiple myeloma, solid tumors, and Paget's disease. Most patients received pamidronate alone, although some patients received pamidronate before zoledronic acid. Renal biopsies, when performed, have shown various glomerulopathies (eg, collapsing glomerulopathy, focal segmental 
glomerulosclerosis) or toxic acute tubular necrosis. In one case series of six patients with renal failure associated with zoledronic acid, it is interesting to note that the renal biopsy results showed toxic acute tubular necrosis without evidence of glomerular damage. ${ }^{18}$ However, all of the six patients had been treated with pamidronate before zoledronic acid. After drug discontinuation, renal function usually improves but may not return to baseline levels.

It is essential that physicians infuse pamidronate $90 \mathrm{mg}$ at a rate no faster than 2 hours or zoledronic acid at a rate no faster than 15 minutes every 3 to 4 weeks. Physicians should not attempt to shorten the infusion time, increase the dose, or reduce the dose interval.

The Update Committee recommends that serum creatinine should be monitored before each dose of pamidronate or zoledronic acid. Serum calcium, electrolytes, phosphate, magnesium, and hematocrit/hemoglobin should also be monitored regularly. The US Food and Drug Administration-approved labeling provides no guidance on time intervals for blood chemistry assessment, but it is specific on pretreatment creatinine measurement. The Update Committee's recommendation is consistent with the current US Food and Drug Administration-approved guidelines in the pamidronate and zoledronic acid package inserts. The Update Committee recognizes that it may be difficult or inconvenient for some clinics to obtain results of renal function tests before pamidronate or zoledronic acid administration. However, the Update Committee recommends that the US Food and Drug Administration-approved monitoring guidelines be followed.

\section{Duration of Therapy}

2007 recommendation. A single randomized clinical trial has shown no benefit of monthly bisphosphonates after tandem stem-cell transplantation. ${ }^{2}$ There was no difference in the proportion of skeletal events in the pamidronate-containing regimens (21\% and $18 \%)$ compared with no maintenance (24\%) after 29 months of follow-up. Given these data and the best clinical opinion of the Update Committee, we suggest that therapy with bisphosphonates be administered monthly for a period of 2 years. (The trial by Attal et $\mathrm{al}^{2}$ suggests 1 year if the patient is in a complete response or near complete response after a tandem stem-cell transplantation.) At 2 years, physicians should seriously consider stopping bisphosphonates in patients with responsive or stable disease, but their further use is at the discretion of the treating physician. There are no data to support a more precise recommendation for duration of bisphosphonate therapy in this group of patients. For those patients in whom bisphosphonates are withdrawn after 2 years, the drug should be resumed on relapse with new-onset skeletal-related events.

Literature update and discussion. Since the original guideline was published, a single randomized clinical trial has been published that relates to the question of the duration of bisphosphonate therapy in patients with multiple myeloma. Attal et $\mathrm{al}^{2}$ conducted a randomized clinical trial to evaluate the role of thalidomide and pamidronate as maintenance therapy in multiple myeloma patients who had undergone tandem stem-cell transplantation. Two months after completing high-dose therapy, 597 patients ( $<65$ years old) were randomly assigned to one of the following three arms: no maintenance therapy, pamidronate (intravenous infusion of $90 \mathrm{mg}$ at 4 -week intervals), or pamidronate plus thalidomide. Groups were compared in terms of response rates; 3-year risk of skeletal events; and event-free, relapsefree, and overall survival. The proportions of patients with skeletalrelated events at 3 years were 24\%,21\%, and 18\% for patients administered no maintenance therapy, pamidronate, and pamidronate plus thalidomide, respectively $(P=.40)$. Survival without skeletal events did not differ among the three treatment groups $(P=.20)$.

\section{Myeloma Patients With Osteopenia Based on Normal Plain Radiograph or Bone Mineral Density Measurements}

2007 recommendation. It is reasonable to start intravenous bisphosphonates in multiple myeloma patients with osteopenia but no radiographic evidence of lytic bone disease. Note, patients with nonlytic lesions have been included in selected trials but have not been the primary focus of the trial or of sufficient number to be separately analyzed.

Literature update. There is no change from the original guideline recommendation. No relevant additional data on this topic were identified from a review of the literature published since 2002 .

\section{Patients With Solitary Plasmacytoma or Smoldering or Indolent Myeloma Without Documented Lytic Bone Disease}

2007 recommendation. Starting bisphosphonate therapy in patients with solitary plasmacytoma or smoldering (asymptomatic) or indolent myeloma is not recommended.

Literature update and discussion. There is no change from the original guideline recommendation. The literature search identified a single relevant study. In this clinical trial, ${ }^{4} 90$ patients with untreated stage I or II myeloma were randomly assigned to either receive or not receive intravenous pamidronate for 1 year. After a median follow-up time of 51 months, disease had progressed in $25 \%$ of patients randomly assigned to pamidronate and in $26.8 \%$ of the controls. The median time to progression was 16.0 months in patients who received pamidronate and 17.4 months in controls. Of the 21 patients who required therapy, skeletal events developed in $82 \%$ of controls and in $40 \%$ of the treated patients $(P<.01)$. The authors concluded that the administration of pamidronate may decrease the development of skeletal events. Pamidronate did not reduce the rate of or the time to disease progression. ${ }^{4}$

\section{Patients With Monoclonal Gammopathy of Undetermined Significance}

2007 recommendation. Starting bisphosphonate therapy in patients with monoclonal gammopathy of undetermined significance is not recommended.

Literature update. There is no change from the original guideline recommendation. No relevant additional data on the use of bisphosphonates for patients with monoclonal gammopathy of undetermined significance were identified from a review of the literature published since 2002 .

\section{Biochemical Markers}

2007 recommendation. The use of the biochemical markers of bone metabolism to monitor bisphosphonate use is not suggested for routine care because of a lack of prospective studies validating such an approach.

Literature update and discussion. There is no change from the original guideline recommendation. The literature search conducted for the update identified two studies of note. A study of 441 patients with prostate cancer, lung cancer, and other solid tumors reported that elevated levels of N-telopeptide (NTX) were a stronger prognostic 
indicator of negative outcomes than bone-specific alkaline phosphatase levels. Patients with high NTX levels had an increased risk of skeletal-related events and disease progression and shorter survival. ${ }^{21}$ In a second study, ${ }^{22}$ urinary measurements of NTX and serum bone alkaline phosphatase were obtained in 1,824 bisphosphonate-treated patients with breast cancer, prostate cancer, non-small-cell lung cancer, other solid tumors, or multiple myeloma. Patients with high and moderate NTX levels had a two-fold increase in their risk of skeletal complications and disease progression compared with patients with low NTX levels $(P<.001)$. Bone alkaline phosphatase showed some correlation with a risk of negative clinical outcomes. ${ }^{22}$ Although the results of these studies are interesting, at this time, the use of these markers should only be practiced within research protocols and have no role in routine care.

\section{Role in Pain Control Secondary to Bony Involvement}

2007 recommendation. Intravenous pamidronate or zoledronic acid is recommended for patients with pain caused by osteolytic disease and as an adjunctive treatment for patients receiving radiation therapy, analgesics, or surgical intervention to stabilize fractures or impending fractures.

Literature update and discussion. There is no change from the original guideline recommendation. The literature review identified a Cochrane Collaboration systematic review on the topic of bisphosphonates for the relief of pain secondary to metastases that was conducted since the original guideline was published in 2002. Wong and Wiffen ${ }^{9}$ identified 30 randomized controlled studies (with a total of 3,682 patients) that investigated the effectiveness of bisphosphonates for pain relief in patients with painful bony metastases from any primary neoplasms (including breast cancer, multiple myeloma, prostate cancer, and any primary cancer site). There were few studies with available data for any of the outcomes considered. Wong and Wiffen ${ }^{9}$ grouped studies by primary disease site using the outcome of the proportion of patients with pain relief within 12 weeks of bisphosphonate therapy. Using this outcome criterion, just one trial of multiple myeloma patients was identified. ${ }^{23}$ The authors concluded that the small number of studies across primary diseases sites did not permit conclusions concerning the relative effectiveness of bisphosphonates. The Expert Panel recommendation from the original (2002) guideline, based on the trial by Berenson et $\mathrm{al}^{23}$ and panel consensus, remains in effect.

\section{Safety and Adverse Effects: ONJ}

NOTE. The topic of ONJ as an adverse effect is new to the guideline.

2007 recommendation. ONJ is an uncommon but potentially serious complication of intravenous bisphosphonates. The Update Committee agrees with the recommendations described in the revised US Food and Drug Administration label for zoledronic acid and pamidronate, Dear Doctor letters, a white paper, and various position papers or statements. All cancer patients should receive a comprehensive dental examination and appropriate preventive dentistry before bisphosphonate therapy. Active oral infections should be treated, and sites at high risk for infection should be eliminated. While on therapy, patients should maintain excellent oral hygiene and avoid invasive dental procedures, if possible.

Review of the literature and discussion. ONJ is an uncommon but potentially serious complication of intravenous bisphosphonate therapy in cancer patients. ${ }^{24-28}$ The first spontaneous reports of ONJ in cancer patients treated with bisphosphonates were received by the US
Food and Drug Administration in 2002, and the first published reports of ONJ appeared in the literature in 2003. More than 300 cases of ONJ associated with intravenous bisphosphonate use in patients with cancer have been reported in the medical and dental literature. ${ }^{29}$ Novartis (East Hanover, NJ) has received 2,400 adverse event reports of ONJ and/or osteomyelitis of the jaw. ${ }^{30}$ In response to growing concerns over ONJ, the manufacturer revised the zoledronic acid and pamidronate package inserts in late 2003 and again in 2004. In addition, Novartis issued Dear Doctor letters concerning ONJ to physicians in September 2004 and to dentists in May 2005 and supported the development of a white paper on ONJ based on an expert multidisciplinary panel, which was distributed to health care professionals in June 2004 and later published. ${ }^{31}$

On the basis of several observational studies, the risk of $\mathrm{ONJ}$ in cancer patients treated with intravenous bisphosphonates ranges from $1 \%$ to $11 \%$, depending on the specific bisphosphonate, bisphosphonate dose, duration of treatment, and dental history. ${ }^{1,32-36}$ However, these figures should be considered estimates because the baseline risk of $\mathrm{ONJ}$ in cancer patients not receiving intravenous bisphosphonates is not known. In a retrospective study of 4,000 cancer patients treated with intravenous bisphosphonate therapy at M.D. Anderson Cancer Center, 33 patients $(0.83 \%)$ with ONJ were identified. ${ }^{35}$ The low incidence reported in that series may be related to the inclusion of many patients who received only a few courses of bisphosphonates (median total dose of pamidronate and zoledronic acid was approximately 180 and $12 \mathrm{mg}$, respectively). The International Myeloma Foundation conducted a Web-based survey of 1,203 patients to assess the risk factors for ONJ. ${ }^{34}$ Sixty-two patients (6.8\%) with myeloma had ONJ, and an additional 54 had suspicious findings; 13 patients (4.3\%) with breast cancer had ONJ, and 23 had suspicious findings. The most reliable estimates of the incidence of bisphosphonateassociated ONJ are based on four cohort studies of cancer patients receiving intravenous bisphosphonates (Table 3). ${ }^{1,32,33,36}$ Each study had an adequate sample size and duration of exposure. The reported incidence of bisphosphonate-associated ONJ ranged from 3.5\% to $11 \%$ in those studies.

The role of bisphosphonates in the etiology of ONJ is not clear because of the presence of other risk factors for ONJ, including previous or concomitant use of chemotherapy, radiotherapy, and corticosteroids. Although ONJ can occur spontaneously, most cases occur in patients after a tooth extraction or other invasive dental procedure. $^{24,29,32-34}$ The type and duration of exposure to (or total dose of) bisphosphonate also seem to be important risk factors. Zoledronic acid has been associated with a higher risk of ONJ than pamidronate (or pamidronate followed by zoledronic acid) in several observational studies (Table 3). ${ }^{1,32-35}$ With follow-up at 36 months, the cumulative incidence of ONJ was $10 \%$ in patients receiving zoledronic acid only compared with $4 \%$ in patients receiving pamidronate with or without zoledronic acid $(P=.002) .{ }^{34}$ Zoledronic acid was also associated with a higher cumulative hazard of ONJ (compared with pamidronate) in two prospective cohort studies. In another prospective cohort study, the relative risk of ONJ associated with zoledronic acid was 9.5 compared with pamidronate alone $(P=.042)$ and 4.5 compared with pamidronate plus zoledronic acid $(P=.018){ }^{1}$ The risk of ONJ seems to increase with time of exposure. ${ }^{24,32-36}$ In prospective cohort studies, the cumulative hazard of ONJ increased from $1 \%$ after 12 months of treatment to up to $11 \%$ at 4 years in one 


\begin{tabular}{|c|c|c|c|c|c|c|}
\hline \multirow[b]{2}{*}{ Study } & \multirow[b]{2}{*}{ Study Design } & \multirow[b]{2}{*}{ Patient Population } & \multicolumn{2}{|c|}{$\begin{array}{l}\text { Duration of } \\
\text { Exposure } \\
\text { (months) }\end{array}$} & \multirow[b]{2}{*}{ Incidence of ONJ (\%) } & \multirow{2}{*}{$\begin{array}{c}\text { Effect of Bisphosphonate or Duration } \\
\text { of Therapy }\end{array}$} \\
\hline & & & Median & Range & & \\
\hline Bamias et $\mathrm{al}^{32}$ & Prospective cohort & $\begin{array}{l}252 \text { patients: } 111 \mathrm{MM} \text {, } \\
\quad 70 \mathrm{BC}, 46 \mathrm{PC}\end{array}$ & 20 & $4-86$ & $\begin{array}{l}\text { Overall: } 6.7 ; \text { MM: } 9.9, \\
\text { BC: } 2.9, \text { PC: } 6.5\end{array}$ & $\begin{array}{l}\text { All patients with ONJ received ZA; cumulative } \\
\text { hazard of ONJ was higher with ZA } \\
(P<.001) \text {; cumulative hazard of ONJ } \\
\text { increased from }>1 \% \text { after } 12 \text { months to } \\
11 \% \text { at } 4 \text { years }\end{array}$ \\
\hline Dimopoulos et $\mathrm{al}^{33}$ & Prospective cohort & 202 patients with MM & 29 & $4-123$ & 7.4 & $\begin{array}{l}14 \text { of } 15 \text { patients with ONJ received ZA; } \\
\text { cumulative hazard of ONJ was higher with } \\
\text { ZA }(P=.001) \text {; cumulative hazard of ONJ } \\
\text { increased from }>1 \% \text { after } 12 \text { months to } \\
13 \% \text { at } 4 \text { years }\end{array}$ \\
\hline Zervas et al ${ }^{1}$ & Prospective cohort & $\begin{array}{l}303 \text { patients with MM; } \\
49 \text { did not receive } \\
\text { ZA or } P\end{array}$ & 24 & $4-120$ & $\begin{array}{l}11 \vee 0 \text { in patients who } \\
\text { did not receive } Z A \\
\text { or } P\end{array}$ & $\begin{array}{l}28 \text { of } 29 \text { patients with ONJ received ZA; } \\
\text { relative risk of ONJ with ZA was } 9.5 v \\
P \text { alone and } 4.5 \vee P+Z A(P<.05)\end{array}$ \\
\hline Tosi et al ${ }^{36}$ & Retrospective cohort & 259 patients with MM & 10 & $4-35$ & 3.5 & $\begin{array}{l}\text { All patients received ZA; risk of ONJ was } \\
6.6 \% \text { after } 24 \text { months }\end{array}$ \\
\hline
\end{tabular}

study $^{32}$ and from $1 \%$ at 12 months to $6 \%$ at 36 months and to $13 \%$ at 48 months in another study. ${ }^{33}$

Several groups and organizations have developed or issued recommendations, position papers, or statements regarding bisphosphonateassociated ONJ. In 2004, Novartis assembled an expert multidisciplinary panel to review the literature and clinical evidence, identify risk factors for ONJ, and develop clinical guidelines for the prevention, early diagnosis, management, and multidisciplinary treatment of ONJ. The panel's recommendations were distributed as a white paper at the 2004 Annual ASCO Meeting and later published. ${ }^{31}$ More recently, the American Academy of Oral Medicine and the American Academy of Oral and Maxillofacial Pathology have published position papers, ${ }^{27,29}$ and the American Association of Endodontists has issued a position statement. $^{37}$ All of these documents agree that prevention of bisphosphonate-associated $\mathrm{ONJ}$ is the best approach to the management of this complication. The Update Committee agrees with the recommendations described in the revised US Food and Drug Administration label for zoledronic acid and pamidronate, Dear Doctor letters, white paper, and various position papers or statements. All cancer patients should receive a comprehensive dental examination and appropriate preventive dentistry before bisphosphonate therapy. Active oral infections should be treated, and sites at high risk for infection should be eliminated. While on therapy, patients should maintain excellent oral hygiene and avoid invasive dental procedures, if possible.

\section{Other Nonrenal Adverse Effects}

The safety and frequency of other nonrenal adverse events with zoledronic acid seem to be similar to pamidronate. The adverse events were well characterized in the pamidronate versus placebo trial, ${ }^{38}$ zoledronic acid versus placebo trials, ${ }^{5,11}$ and the pamidronate versus zoledronic acid trials. ${ }^{10,39}$ The incidence of most adverse effects in patients treated with pamidronate was similar to that observed in the placebo group. Transient myalgias, arthralgias, and flu-like symptoms with fever tend to occur more often in patients treated with pamidronate or zoledronic acid than placebo. ${ }^{5,11,38,40}$ These symptoms usually occur only after the first and/or second infusion of pamidronate and are not an indication to discontinue drug treatment. Ocular adverse effects from pamidronate are relatively rare but well-recognized complications that were first reported in $1994 .^{41}$ An updated review of case reports found 17 patients with unilateral scleritis and one patient with bilateral scleritis, usually within 6 hours to 2 days after intravenous pamidronate. Six patients had positive rechallenge testing, with the scleritis occurring again after a repeat drug exposure. ${ }^{42}$ Ocular adverse effects have been reported with zoledronic acid ${ }^{43}$ and other bisphosphonates. ${ }^{44}$

\section{AUTHORS' DISCLOSURES OF POTENTIAL CONFLICTS OF INTEREST}

Although all authors completed the disclosure declaration, the following authors or their immediate family members indicated a financial interest. No conflict exists for drugs or devices used in a study if they are not being evaluated as part of the investigation. For a detailed description of the disclosure categories, or for more information about ASCO's conflict of interest policy, please refer to the Author Disclosure Declaration and the Disclosures of Potential Conflicts of Interest section in Information for Contributors.

Employment: N/A Leadership: N/A Consultant: Robert A. Kyle, Novartis; David G. Roodman, Amgen, Novartis, Merck, Millennium; Kenneth Anderson, Novartis, Celgene, Millenium Stock: N/A Honoraria: Robert A. Kyle, Novartis; Gary C. Yee, Novartis; David G. Roodman, Amgen, Novartis, Merck, Millennium; Kenneth Anderson, Novartis, Millenium, Celgene Research Funds: Kenneth Anderson, Millenium, Celgene Testimony: N/A Other: N/A

\section{AUTHOR CONTRIBUTIONS}

Administrative support: Mark R. Somerfield

Collection and assembly of data: Mark R. Somerfield

Data analysis and interpretation: Robert A. Kyle, Gary C. Yee, Mark R. Somerfield, Patrick J. Flynn, Susan Halabi, Sundar Jagannath, Robert Z. Orlowski, David G. Roodman, Kenneth Anderson 
Manuscript writing: Robert A. Kyle, Gary C. Yee, Mark Somerfield, Patrick J. Flynn, Susan Halabi, Sundar Jagannath, Robert Z. Orlowski, David G. Roodman, Patricia Twilde, Kenneth Anderson
Final approval of manuscript: Robert A. Kyle, Gary C. Yee, Patrick J. Flynn, Susan Halabi, Sundar Jagannath, Robert Z. Orlowski, David G. Roodman, Patricia Twilde, Kenneth Anderson

\section{REFERENCES}

1. Zervas K, Verrou E, Teleioudis Z, et al: Incidence, risk factors and management of osteonecrosis of the jaw in patients with multiple myeloma: $\mathrm{A}$ single-centre experience in 303 patients. $\mathrm{Br} \mathrm{J}$ Haematol 134:620-623, 2006

2. Attal M, Harousseau JL, Leyvraz $S$, et al: Maintenance therapy with thalidomide improves survival in multiple myeloma patients. Blood 108: 3289-3294, 2006

3. Lacy $M Q$, Dispenzieri $A$, Gertz $M A$, et al: Mayo clinic consensus statement for the use of bisphosphonates in multiple myeloma. Mayo Clin Proc 81:1047-1053, 2006

4. Musto P, Falcone A, Sanpaolo G, et al: Pamidronate reduces skeletal events but does not improve progression-free survival in early-stage untreated myeloma: Results of a randomized trial. Leuk Lymphoma 44:1545-1548, 2003

5. Rosen LS, Gordon D, Kaminski M, et al: Long-term efficacy and safety of zoledronic acid compared with pamidronate disodium in the treatment of skeletal complications in patients with advanced multiple myeloma or breast carcinoma: A randomized, double-blind, multicenter, comparative trial. Cancer 98:1735-1744, 2003

6. Imrie K, Stevens A, Makarski J, et al: The role of bisphosphonates in the management of skeletal complications for patients with multiple myeloma: A clinical practice guideline. http://www.cancercare.on .ca/pdf/pebc6-4f.pdf

7. Djulbegovic B, Wheatley K, Ross J, et al: Bisphosphonates in multiple myeloma. The Cochrane Collaboration. http://www.mrw.interscience .wiley.com/cochrane/clsysrev/articles/CD003188/ frame.html

8. Ross JR, Saunders Y, Edmonds PM, et al: A systematic review of the role of bisphosphonates in metastatic disease. Health Technol Assess 8:1-176, 2004

9. Wong R, Wiffen PJ: Bisphosphonates for the relief of pain secondary to bone metastases. The Cochrane Collaboration. http://www.mrw.interscience .wiley.com/cochrane/clsysrev/articles/CD002068/ frame.html

10. Rosen LS, Gordon D, Kaminski M, et al: Zoledronic acid versus pamidronate in the treatment of skeletal metastases in patients with breast cancer or osteolytic lesions of multiple myeloma: A phase III, double-blind, comparative trial. Cancer J 7:377-387, 2001

11. Saad F, Gleason DM, Murray $R$, et al: A randomized, placebo-controlled trial of zoledronic acid in patients with hormone-refractory metastatic prostate carcinoma. J Natl Cancer Inst 94:14581468,2002

12. Schran $\mathrm{H}$ : Use of Zometa in Patients With Renal Impairment. Basel, Switzerland, Novartis Pharmaceuticals, 2005

13. Guarneri V, Donati $S$, Nicolini $M$, et al: Renal safety and efficacy of i.v. bisphosphonates in patients with skeletal metastases treated for up to 10 years. Oncologist 10:842-848, 2005
14. Bujanda DA, Sarmiento UB, Suárez MAC, et al: Assessment of renal toxicity and osteonecrosis of the jaws in patients receiving zoledronic acid for bone metastasis. Ann Oncol 18:556-560, 2007

15. Barri YM, Munshi NC, Sukumalchantra S, et al: Podocyte injury associated glomerulopathies induced by pamidronate. Kidney Int 65:634-641, 2004

16. Chang JT, Green L, Beitz J: Renal failure with the use of zoledronic acid. N Engl J Med 349:16761679, 2003

17. Kunin M, Kopolovic J, Avigdor A, et al: Collapsing glomerulopathy induced by long-term treatment with standard-dose pamidronate in a myeloma patient. Nephrol Dial Transplant 19:723-726, 2004

18. Markowitz GS, Fine PL, Stack JI, et al: Toxic acute tubular necrosis following treatment with zoledronate (Zometa). Kidney Int 64:281-289, 2003

19. Munier A, Gras V, Andrejak $M$, et al: Zoledronic acid and renal toxicity: Data from French adverse effect reporting database. Ann Pharmacother 39:1194-1197, 2005

20. Sauter M, Julg B, Porubsky S, et al: Nephroticrange proteinuria following pamidronate therapy in a patient with metastatic breast cancer: Mitochondrial toxicity as a pathogenetic concept? Am J Kidney Dis 47:1075-1080, 2006

21. Brown JE, Cook RJ, Major $P$, et al: Bone turnover markers as predictors of skeletal complications in prostate cancer, lung cancer, and other solid tumors. J Natl Cancer Inst 97:59-69, 2005

22. Coleman RE, Major P, Lipton A, et al: Predictive value of bone resorption and formation markers in cancer patients with bone metastases receiving the bisphosphonate zoledronic acid. J Clin Oncol 23:4925-4935, 2005

23. Berenson JR, Lichtenstein $A$, Porter $L$, et al: Efficacy of pamidronate in reducing skeletal events in patients with advanced multiple myeloma: Myeloma Aredia Study Group. N Engl J Med 334:488493, 1996

24. Badros A, Weikel D, Salama A, et al: Osteonecrosis of the jaw in multiple myeloma patients: Clinical features and risk factors. J Clin Oncol 24: 945-952, 2006

25. Bagan JV, Murillo J, Jimenez Y, et al: Avascular jaw osteonecrosis in association with cancer chemotherapy: Series of 10 cases. J Oral Pathol Med 34:120-123, 2005

26. Marx RE, Sawatari $Y$, Fortin $M$, et al: Bisphosphonate-induced exposed bone (osteonecrosis/osteopetrosis) of the jaws: Risk factors, recognition, prevention, and treatment. J Oral Maxillofac Surg 63:1567-1575, 2005

27. Migliorati CA, Schubert MM, Peterson DE, et al: Bisphosphonate-associated osteonecrosis of mandibular and maxillary bone: An emerging oral complication of supportive cancer therapy. Cancer 104:83-93, 2005

28. Ruggiero SL, Mehrotra B, Rosenberg TJ, et al: Osteonecrosis of the jaws associated with the use of bisphosphonates: A review of 63 cases. J Oral Maxillofac Surg 62:527-534, 2004

29. Woo SB, Hellstein JW, Kalmar JR: Narrative [corrected] review: Bisphosphonates and osteone- crosis of the jaws. Ann Intern Med 144:753-761, 2006

30. Kuehn BM: Reports of adverse events from bone drugs prompt caution. JAMA 295:2833-2836, 2006

31. Ruggiero S, Gralow J, Marx RE, et al: Practical guidelines for the prevention, diagnosis, and treatment of osteonecrosis of the jaw in patients with cancer. J Oncol Pract 2:7-14, 2006

32. Bamias A, Kastritis E, Bamia C, et al: Osteonecrosis of the jaw in cancer after treatment with bisphosphonates: Incidence and risk factors. J Clin Oncol 23:8580-8587, 2005

33. Dimopoulos MA, Kastritis E, AnagnostopouIos A, et al: Osteonecrosis of the jaw in patients with multiple myeloma treated with bisphosphonates: Evidence of increased risk after treatment with zoledronic acid. Haematologica 91:968-971, 2006

34. Durie BG, Katz M, Crowley J: Osteonecrosis of the jaw and bisphosphonates. N Engl J Med 353:99-102, 2005

35. Hoff AO, Toth B, Altundag K, et al: Osteonecrosis of the jaw in patients receiving intravenous bisphosphonate therapy. Presented at the 27th Annual Meeting of the American Society for Bone and Mineral Research, Nashville, TN, September 22-26, 2005

36. Tosi $P$, Zamagni $E$, Cangini $D$, et al: Osteonecrosis of the jaws in newly diagnosed multiple myeloma patients treated with zoledronic acid and thalidomide-dexamethasone. Blood 108:3951-3952, 2006

37. American Association of Endodontists Position Statement: Endodontic implications of bisphosphonate-associated osteonecrosis of the jaws. http://www.stateendodontics.com/articles/ bisphosonatesstatement.pdf

38. Hortobagyi GN, Theriault RL, Porter $\mathrm{L}$, et al: Efficacy of pamidronate in reducing skeletal complications in patients with breast cancer and lytic bone metastases: Protocol 19 Aredia Breast Cancer Study Group. N Engl J Med 335:1785-1791, 1996

39. Berenson JR, Rosen LS, Howell A, et al: Zoledronic acid reduces skeletal-related events in patients with osteolytic metastases. Cancer 91: 1191-1200, 2001

40. Coukell AJ, Markham A: Pamidronate: A review of its use in the management of osteolytic bone metastases, tumour-induced hypercalcaemia and Paget's disease of bone. Drugs Aging 12:149168,1998

41. Macarol V, Fraunfelder FT: Pamidronate disodium and possible ocular adverse drug reactions. Am J Ophthalmol 118:220-224, 1994

42. Fraunfelder FW, Fraunfelder FT, Jensvold B: Scleritis and other ocular side effects associated with pamidronate disodium. Am J Ophthalmol 135: 219-222, 2003

43. Durnian JM, Olujohungbe A, Kyle G: Bilateral acute uveitis and conjunctivitis after zoledronic acid therapy. Eye 19:221-222, 2005

44. Fraunfelder FW, Fraunfelder FT: Bisphosphonates and ocular inflammation. N Engl J Med 348: 1187-1188, 2003 


\section{Acknowledgment}

The Update Panel expresses its gratitude to Sandra Horning, MD, and Alan Lichtin, MD, and members of the American Society of Clinical Oncology Health Services Committee for their reviews of earlier drafts of this guideline update.

\section{Appendix 1}

\section{American Society of Clinical Oncology Bisphosphonates in Multiple Myeloma Guideline Update Panel}

Kenneth Anderson, MD, Co-Chair, Dana-Farber Cancer Institute; Robert A. Kyle, MD, Co-Chair, Mayo Clinic; Patrick J. Flynn, MD, Minnesota Oncology Hematology P.A.; Sundar Jagannath, MD, St Vincent's Comprehensive Cancer Center; Susan Halabi, PhD, Duke University Medical Center; Robert Orlowski, MD, PhD, University of North Carolina at Chapel Hill; David Roodman, MD, VA Pittsburgh Healthcare System; Patricia Twilde, Patient Representative; and Gary C. Yee, Pharm D, University of Nebraska Medical Center.

\section{Appendix 2}

For the 2007 update, a methodology similar to that applied in the original American Society of Clinical Oncology (ASCO) practice guideline for use of bisphosphonates in multiple myeloma was used. Pertinent information published from 2002 to 2007 was reviewed to address each of the original guideline questions and the new topic of osteonecrosis of the jaw. The Medline database (National Library of Medicine, Bethesda, MD) was searched to identify relevant information from published randomized clinical trials, systematic reviews, meta-analyses, and practice guidelines for this update. A series of searches was conducted using the medical subject headings or text words "multiple myeloma" and "bisphosphonates" and variants thereof. Targeted searches using broad inclusion criteria were conducted to identify relevant articles related to osteonecrosis of the jaw. Search results were limited to human studies and English-language articles; editorials, letters, and commentaries were excluded from consideration. The Cochrane Library was searched for available systematic reviews and meta-analyses with words "biphosphonates," "bisphosphonates," and "diphosphonates." Directed searches based on the bibliographies of primary articles were also performed. Finally, Update Committee members and ASCO staff contributed articles from their personal collections. Update Committee members reviewed the resulting abstracts and titles that corresponded to their assigned section. 